Gabriele ZIETHEN

„SKLAVENRACHE“ ODER DIE RETTUNG DES AMHARENKNABEN. EIN WENIG
BEKANNTER TEXT VON KARL MAY

Um 1889 wähnte sich das literarische Ich des Autors Karl May (geb. 25.02.1842 in Ernstthal [Sachsen], gest. 30.03.1912 in Radebeul bei Dresden) - oder war er es vielleicht sogar leibhaftig und persönlich? - unter den Besuchern einer sog. „Völkerwiese“, wie sie besonders auch unter dem Namen der Hagenbeck'schen Völkerschauen berühmt war. Schon sehr bald geht unser Besucher mit einer Gruppe publikumswirksam dargebotener Somalis ,auf Tuchfühlung“, so wie wir sein literarisches alter ego in Gestalt des Old Shatterhand und Kara ben Nemsi im Verlauf zahlreicher Abenteuererzählungen während des in fremden Ländern angesiedelten Geschehens kennen. Die Eingeborenen - Männer, Frauen und Kinder werden detailliert beschrieben, man bietet ihnen Obst, Zigarren und kleine Geldstücke an, und freut sich über die naive Dankbarkeit der so Gefütterten und Beschenkten.

Der Autor, von Beruf ursprünglich Lehrer ${ }^{1}$, erläutert in seinem Text „Das Straussenreiten der Somal“, erschienen in der Zeitschrift „Der gute Kamerad“, die kulturelle Relevanz der „Völkerwiesen“ mit den folgenden Bemerkungen:

„Seit Deutschland in die Reihe der Kolonialstaaten getreten ist, hat sich unser schon vorher so reges Interesse für fremde Völkerschaften in der Weise vergrößert, daß es fast in jeder bedeutenderen Stadt unsres lieben Vaterlandes eine sogenannte »Völkerwiese« gibt, das heißt, einen öffentlichen Ort, an dem von Zeit zu Zeit die Vertreter der verschiedensten fremden Nationen in der Ausübung ihrer friedlichen und kriegerischen Künste zu beobachten sind. Wir haben Sioux und Pescherähs, Eskimos und Zulus, australische Buschmänner und Singhalesen, Ainos und menschenfressende Neger bei uns gesehen und sie alle in ihrem eigenartigen Thun und Treiben beobachten können. Gegenwärtig nun macht eine Karawane von Somal, welche als Nachbarn des unter deutschem Schutze stehenden Witu-Landes unsre regste Teilnahme erwecken müssen, die

Ausführlich zur Biographie Hermann Wohlgschaft: Große Karl May Biographie. Leben und Werk. Paderborn 1994. 
Runde durch die erwähnten Völkerwiesen, und wer Zeuge ihrer Schaustellungen gewesen ist, wird zu der Ansicht gelangt sein, daß der sogenannte schwarze Erdteil keineswegs nur von bildungsunfähigen Elementen bevölkert ist.“2

Es folgt eine Zusammenfassung ethnographischer und kulturanthropologischer Ansichten, die kompilierend aus den Werken verschiedener Forscher, u.a. von Georges Révoil (1852-1894) ${ }^{3}$, übernommen wurden.

\begin{abstract}
„Der Somali ist Nomad; er verachtet das Handwerk und hält nur Raub und Krieg für seiner würdig; alle Arbeit fällt den Frauen zu. Er ist mutig, tapfer und freiheitsliebend, aber auch streitsüchtig und hinterlistig und aller Ordnung abgeneigt. Die Blutrache wird bis aufs äußerste getrieben und ist die Ursache, daß die einzelnen Stämme sich untereinander durch ewige Fehden aufreiben. Darum haben die meisten Versuche, das Land zu erforschen, zu einem negativen Resultate geführt, und der französische Reisende Revoil sagt mit Recht: »Das einzige bebaute Feld im Somalilande ist der Totenacker.« Ein andrer Forscher fällt folgendes ebenso wahre Urteil: »Der Somaliknabe erhält im siebenten Jahre einen kleinen Speer, bettelt, borgt und stiehlt, bis er Mann geworden ist, und bettelt, borgt und stiehlt dann weiter bis er stirbt." 4
\end{abstract}

Diese Ansichten nun bieten den Hintergrund für den ebenfalls 1889 im gleichen Format der „Gute Kamerad“ unter dem Titel „Sklavenrache“ erschienenen Text.

2 Das Straußenreiten der Somal. In: Der Gute Kamerad. 4. Jg. Nr. 13. S. 175-177. - Berlin, Stuttgart (1889), S. 175-177. In: Der Gute Kamerad. Spemanns Illustrierte KnabenZeitung. [Jahrgangstitel: Der Gute Kamerad. Spemanns Illustriertes Knaben-Jahrbuch]. 4. Jg. Nr. 13. S. 175-177. - Berlin, Stuttgart: W. Spemann (1889). Reprint in: Der Schwarze Mustang. Anhang: Die kleineren »Kamerad«-Erzählungen von Karl May. Einführung von Erich Heinemann. Hamburg: Karl-May-Gesellschaft 1991. Permalink: http://www.zeno.org/nid/20005369363 [Dateneinsicht: 05.03.2018]

Georges Révoil: Voyages au cap des Aromates (Afrique orientale) 1877-1878. Illustrations de Ferdinandus et G. Bellenger; cartes gravées par Erhard d'après les croquis et documents de l'auteur. Paris, E. Dentu, 1880 [Nachdr. hrsg. von. Victor Attinger, Paris, 1932]; ders.: La Vallée du Darror; voyage aux pays Çomalis (Afrique orientale) types, scènes, paysages, panoramas hors texte, d'après les photographies et les croquis de l'auteur; publiée par la Société de Géographie de Paris. Paris 1882. - ders.: Dix mois à la côte orientale d'Afrique: la Vallée du Darror et le Cap Guardafui. Paris 1889.

s. Das Straußenreiten der Somal (Anm. 2) S. 176.

Sklavenrache. In: Der Gute Kamerad. 4. Jg. Nr. 3. S. 35-37. -Berlin, Stuttgart (1889), S. 35-37. In: Der Gute Kamerad. Spemanns Illustrierte Knaben-Zeitung. [Jahrgangstitel: Der Gute Kamerad. Spemanns Illustriertes Knaben-Jahrbuch]. 4. Jg. Nr. 3. S. 35-37. - Berlin, Stuttgart: W. Spemann (1889). Reprint in: Der Schwarze Mustang. Anhang: Die kleineren 
Dieser Text fällt zeitlich mit der Werkphase des Orientzyklus zusammen, unterscheidet sich aber noch erheblich von den eher geläufigen Romantexten. Stilistisch jedoch eher schwerfällig daherkommend, fällt inhaltlich die harte Schilderung besonderer Grausamkeiten auf. Seitens der Literaturforschung wird dies im Zusammenhang mit Karl Mays eigener Aufarbeitung seiner als grausam empfundenen langjährigen Haftstrafen, welche die Folge mehrerer Betrugsdelikte waren, erklärt (s. Anm. 1 u. Anm. 20).

Betrachtet man jedoch den Entwicklungsweg des Autors in seiner Gesamtheit, so liegen zwischen diesem Text und dem auf Ausgleich, Einsicht und durchaus auch altersmilder pazifistischer Weisheit bedachten, heute immer noch lesenswerten, Alterswerk nach 1905 Welten. Diesen Spannungsbogen gilt es zu entdecken.-

Den Blick von der kolonialen „Völkerwiese“ jetzt dem unwegsamen Gelände am Horn von Afrika zuwendend, sehen wir in unwegsamen Gelände eine Menschenkarawane, bestehend aus Somalis und Amharen, ihrem Schicksal begegnen:

KARL MAY, SKLAVENRACHE. - „Es war im August, also während der Sommerregenzeit, als eine kleine Karawane langsam im Halimalathale herabgestiegen kam. Sie bestand aus etwa fünfzig männlichen und weiblichen Amharasklaven und zwanzig bewaffneten Treibern, welche der Sippe der Schibril Abokr-Somal angehörten. Diese letzteren pflegen die Sklaven in Harar abzuholen und bis diesseits des genannten Thales zu bringen, wo ihnen die Isa-Somal die menschliche Ware abnehmen, um dieselbe an die Küste zu transportieren. Die Amhara waren mit Stricken aneinander gefesselt. Ihre Rasse ist eine der heitersten und gewecktesten; diesen ihrer Heimat mit Gewalt entrissenen Leuten aber war das keineswegs anzusehen. Die Isa sind die häßlichsten und grausamsten unter den Somal; die Sklaven schritten also einer Verschlimmerung ihres schon bisher so herben Schicksals entgegen.

Das Thal mündete auf eine Ebene, auf welcher der Regen einen frischen Graswuchs hervorgelockt hatte. Auch die dastehenden Wababäume, welche das Pfeilgift der Somal liefern, hatten ihre immergrünen Blätter heller gefärbt. Unter ihren Kronen stand ein sonst verlassenes Hüttendorf, von dem eine Isa-Schar Besitz ergriffen hatte, um da die Schibril Abokr mit den Sklaven zu erwarten.

Als der Zug derselben sich zeigte, rannten sie ihm schreiend und die Waffen schwingend entgegen. Die Begrüßung fand in gewohnter lärmender Weise statt und dann wurden die

»Kamerad«-Erzählungen von Karl May. Einführung von Erich Heinemann. Hamburg: Karl-May-Gesellschaft 1991. Permalink: http://www.zeno.org/nid/20005369347. 


\section{Gabriele ZIETHEN}

Amhara nach dem Innern des Dorfes geführt, welches mit hohen, stacheligen Dornzäunen umgeben war. Am Eingange derselben stellte man mehrere Wächter auf.

Zunächst begann das Feilschen um den Preis der Sklaven. Jeder derselben wurde genau untersucht und dabei vielfach gepeinigt. Als man sich geeinigt hatte, erfolgte die Bezahlung in Tüchern, Schießpulver und allerlei Eisenwaren. - Darauf sollte der unvermeidliche Schmaus folgen, stark mit rotem Pfeffer gewürztes Schöpsenfleisch [i.e. Hammelfleisch, Anm. G.Z.], welches die Frauen der Isa inzwischen bereitet hatten.

Ein Isaknabe hatte unter den Gefangenen zwei in seinem Alter stehende Brüder bemerkt, welche sich ermüdet an die hohe, stolze Gestalt ihres Vaters gelehnt hatten. Einer Regung seines Herzens folgend, trat er zu ihnen, legte dem einen die Hand auf den Kopf und sagte laut, so daß alle es hörten:

»Ich bin dein Abban (Beschützer), und du bist frei.«

Nach der Sitte des Landes war der junge Amhara nun kein Sklave mehr. Schon erhob der Isaknabe den Arm, um auch den andern von der Knechtschaft zu befreien, da sprang der Anführer der Isa herbei, riß ihn zurück und schrie:

»Bist du toll? Haben wir diese Hunde bezahlt, um sie nun frei zu geben? Das Wort eines Isa gilt, selbst wenn er ein Kind ist. Dieser Amharabube gehört also nicht mehr zu den Sklaven; aber da wir ihn nicht verkaufen können, wollen wir ihn auch nicht mit uns schleppen!«

Er zog sein langes, zweischneidiges Messer und stieß es dem Amharaknaben in das Herz. Keiner der Somal erschrak über diesen Mord; solche Blutthaten gehören bei ihnen zu den Alltäglichkeiten; aber die Sklaven schrieen vor Entsetzen auf. Der nicht an den Händen gefesselte Vater des Ermordeten stand einen Augenblick lang wie erstarrt, dann entriß er dem im Bereiche seiner Arme stehenden Mörder das Messer, rannte es ihm in die Brust, durchschnitt dann den Strick, der ihn mit seinen Leidensgenossen verband, und stürzte sich, vor Wut brüllend, mitten unter die Somal, deren er mehrere schwer verwundete.

Da der Strick entzwei war, gelang es mehreren Amhara, sich loszumachen. Der Kämpfende sah das; er erkannte, daß er der Übermacht erliegen müsse; darum ergriff er die Flucht und rief den Seinen zu:

»Flieht, mir nach, und dann Rache!«

Es war die höchste Zeit für ihn, denn schon hatte man ihm das Messer entnissen. Er rannte dem Eingange des Dorfes zu, und mehrere Genossen folgten ihm. Aber die dort stehenden Wächter hatten den Lärm gehört; sie waren auf ihrer Hut, und eben als er durch die Lücke des Dornzaunes sprang, stieß ihm einer derselben den Speer durch den Leib. Die andern Flüchtigen sahen das; sie verloren den Mut, getrauten sich nicht weiter und wurden nach kurzer Gegenwehr überwältigt.

Wären die Schibril Abokr schon wieder abgezogen gewesen, so hätte das Ereignis für die Isa einen schlimmen Ausgang nehmen können, da dieselben nicht mehr als fünfzehn Krieger zählten. Sie beschlossen also, die Aufwiegler aufs strengste zu bestrafen. Man sonderte sie von den andern Sklaven, band ihnen die Füße und hielt dann Gericht über sie. Da sie verkauft werden sollten, wollte man ihr Leben schonen; nur der trotzigste von ihnen wurde zum Tode verurteilt, und zwar zu einem sehr grausamen. Man band ihn in eine alte Decke, so daß er sich nicht zu regen vermochte, und sprang dann so lange auf ihm herum, bis ihm alle Rippen und Gelenke zerbrochen waren. In diesem Zustande sollte er liegen bleiben.

Die andern wurden einer nach dem andern an einen verdorrten Stamm gebunden und grausam durchgepeitscht, wobei ihnen die Weiber roten Pfeffer in das von den Ruten 
aufgerissene Fleisch streuten. Hierauf wurde der vorhin begonnene Schmaus fortgesetzt, als ob derselbe durch nichts unterbrochen worden sei. Er währte bis in die Nacht hinein. Am andern Tage brach man auf. Die Schibril Abokr wandten sich zurück, und die Isa nahmen die Richtung nach der Küste, nachdem sie ihren toten Anführer unter einem Steinhaufen begraben hatten. Die toten Amhara, Vater und Sohn, wurden den wilden Tieren überlassen. Der von den Füßen Zermalmte lebte noch; seine Genossen konnten im Weiterziehen noch lange sein Rufen und Wimmern hören.

Die Karawane hatte die Hochebene des Landesinnern hinter sich und passierte nun die Hügelregion, in welcher man zahlreiche Torrents trifft, Flußbetten, die nur während der Regenzeit Wasser führen. $\mathrm{Zu}$ dieser Zeit ist es gefährlich, sich in so einem trockenen Bette aufzuhalten. Geht nämlich oben in den Bergen ein plötzlicher Regen nieder, welcher gewöhnlich einem Wolkenbruche gleicht, so stürzt das Wasser mit Macht vorwärts und reißt alles mit sich fort. Das Wasser kommt wie eine hohe Mauer herangebraust, und nach wenigen Minuten hat sich das vorher trockene, stille Thal in einen brüllenden, verderbenbringenden Strom verwandelt.

Ein solcher trockener Torrent wurde gegen Abend erreicht. Da der letzte Regen hier einiges Wasser zurückgelassen hatte, dessen man zum Trinken bedurfte, so wurde in demselben für die Nacht Halt gemacht. Die Isa zählten, wie bereits gesagt, fünfzehn Krieger; dazu kamen mehrere Frauen, denen bei den Somal selbst während der Reise alle Arbeit obliegt, und der auch schon erwähnte Knabe. Die Sklaven waren wieder an den langen Strick gebunden und mußten auch an demselben schlafen, auch diejenigen, welche gepeitscht worden waren und ihre Schmerzen kaum zu unterdrücken vermochten. Nur der Amharaknabe konnte sich frei bewegen, da anzunehmen war, daß er nicht davonlaufen werde.

Es war eine mondeshelle Nacht, deren Stille nur durch die Stimme des Schakals und den Ruf des Regenpfeifers unterbrochen wurde. Die Gefangenen lagen dicht zusammendrängt, um sich gegenseitig zu erwärmen, da die Nächte hier sehr kühl zu sein pflegen. Die Isa saßen und lagen an einem Feuer, die letzteren schliefen; die ersteren hatten die Sklaven zu bewachen. Es mochte nahe an Mitternacht sein, da erhob sich erst ein leiser Wind, welcher immer stärker wurde. Er war kalt und feucht und kam von den Bergen her. Sein Rauschen war hohl und unheimlich; die Schläfer erwachten davon. Da plötzlich mischte sich in dieses Rauschen ein eigenartiges Brausen, welches näher zu kommen schien; bald war es kein Brausen mehr sondern ein donnerndes Brüllen. Die Amhara kannten als Gebirgsbewohner diesen elementaren Ton. Sie sprangen erschrocken auf.

»Um Gotteswillen, der Schellal kommt! Rettet euch!« rief einer von ihnen.

Schellal heißt Katarakt, Flut, Wassermasse. Die Isa hörten diese Worte und griffen nach ihren Habseligkeiten, um zu fliehen. Vielleicht war es ihnen noch möglich, das steile Ufer zu erklimmen; die am Seile hängenden Amhara mochten immerhin ertrinken. Da aber brüllte einer von diesen, so daß seine Stimme diejenige des herantosenden Wassers übertönte:

»Müssen wir ertrinken, dann sollen sie das auch! Haltet sie fest, haltet sie fest!« Augenblicklich wurden die Isa von den Amhara wie von einer undurchdringlichen Kette umschlossen. Jeder Sklave packte einen seiner Peiniger. Diese letzteren gebrauchten zwar ihre Waffen, aber es war zu spät. Eine hohe, dunkle, tosende Masse schoß heran, ergriff Freund und Feind, hob sie empor und riß sie mit sich fort. 
Von den andern unbeachtet war der Amharaknabe zu dem kleinen Isa gesprungen und hatte ihn gefragt:

»Kannst du schwimmen?«

$»$ Nein.«

»So halte dich an mir fest. Du hast den Bruder und mich retten wollen; dafür rette ich dich!«

Der Schellal ging vorüber, und das Wasser floß ruhiger zwischen den Ufern. Der Mond spiegelte sich in der Flut und beleuchtete zwei Knaben, welche dem Flusse folgten, um zu sehen, ob sich jemand gerettet habe - vergebliches Suchen! Der Strom hatte in die Rache der Sklaven gewilligt und sie samt ihren Herren verschlungen. Nur die beiden Knaben waren dem Verderben entgangen. Sie hielten sich umschlungen und der kleine Isa sagte zu dem Amhara:

»Nun werde ich doch noch dein Abban sein. Wir werden bald Männer meines Stammes treffen, und der beste von ihnen wird dich als Sohn aufnehmen, weil du aus Dankbarkeit mein Leben höher als das deinige geachtet hast. «"

Text-Quelle: Sklavenrache. In: Der Gute Kamerad. 4. Jg. Nr. 3. S. 35-37. - Berlin, Stuttgart (1889), S. 35-37. - In: Der Gute Kamerad. Spemanns Illustrierte Knaben-Zeitung. [Jahrgangstitel: Der Gute Kamerad. Spemanns Illustriertes Knaben-Jahrbuch]. 4. Jg. Nr. 3. S. 35-37. - Berlin, Stuttgart: W. Spemann (1889). Reprint in: Der Schwarze Mustang. Anhang: Die kleineren »Kamerad«-Erzählungen von Karl May. Einführung von Erich Heinemann. Hamburg: Karl-May-Gesellschaft 1991. Permalink: http://www.zeno.org/nid/20005369347. Lizenz: Gemeinfrei. Kategorien: Erzählung. - Text aus:

http://www.zeno.org/Literatur/M/May,+Karl/Einzelne+Erz\%C3\%A4hlungen/Sklavenrache [26.03.2017]

\section{Völkerschau}

Karl Mays Text aus dem Jahr 1889 fällt in eine Zeit, in der das Deutsche Kaiserreich koloniale Interessen in Afrika verfolgte und somit in dem hier interessierenden Zusammenhang auf entsprechende Aktivitäten in der Region der Somaliküste ${ }^{6}$ zwischen 1885 und 1890 (Deutsche Witu-

Zum Einstieg in die umfangreiche Thematik seien die Literatur- und Rezensionsverzeichnisse unter https:/www.hsozkult.de empfohlen. - Hanco Jürgens: Rezension zu: Deutscher Kolonialismus. Fragmente seiner Geschichte und Gegenwart, 14.10.2016 - 14.05.2017 Berlin, in: H-Soz-Kult, 26.11.2016, <www.hsozkult.de/exhibitionreview/id/rezausstellungen-254>. - Jens Jäger: Rezension zu: Bradley Naranch, Geoff Eley (Hrsg.): German Colonialism In A Global Age. Durham 2014, in: H-Soz-Kult, 10.01.2017, <www.hsozkult.de/publicationreview/id/rezbuecher24116>. - Stefanie Michels: Rezension zu: Tanja Bührer: Die Kaiserliche Schutztruppe für Deutsch-Ostafrika. Koloniale Sicherheitspolitik und transkulturelle Kriegführung, 1885 bis 1918. München 2011, in: H-Soz-Kult, 02.03.2012, <www.hsozkult.de/publicationreview/id/rezbuecher-16363. - Michael Mann: Rezension zu: Gwyn Campbell (Hrsg.): The Structure of Slavery in Indian Ocean Africa and Asia. 
Gesellschaft, ab 1887 Deutsche Kolonialgesellschaft) hinzuweisen ist. Um der allgemeinen Bevölkerung die Fremdheit und Buntheit der Völker des afrikanischen Kontinents anschaulich näherzubringen, wurden in Europa sog. „Völkerwiesen“ und „Völkerschauen“ veranstaltet. Menschen, die unter Zwang und falschen Versprechungen aus ihrer Heimat nach Europa verbracht wurden, mußten ihr tägliches Leben in einer lebenden Ausstellung samt Naturinszenierung präsentieren. ${ }^{7}$ Diese Veranstaltungen fanden ein großes Publikum, das oft zum ersten Mal „Fremde“ aus einem anderen Kulturkreis aus nächster Nähe sehen konnte und zumeist den Sinn der kolonialen Aufgaben darin sah, die Überlegenheit der europäischen Kultur zu verdeutlichen und somit die kolonialen Aktivitäten zu begründen. Eng verbunden mit den Völkerschauen war ein reger internationaler Tierhandel nach Europa und den USA ${ }^{8}$.

Somalier und Äthiopier wurden z.B. bei Carl Hagenbeck häufig gezeigt, da diese Völker nicht den in den deutschen Kolonien geltenden Ausfuhr- und Anwerbeverboten zu „Schaustellzwecken“ der Jahre 1891

London 2004 / Campbell, Gwyn (Hrsg.): Abolition and its Aftermath in Indian Ocean Africa and Asia. London 2005, in: Connections. A Journal for Historians and Area Specialists, 18.10.2007, <www.connections.clioonline.net/publicationreview/id/rezbuecher-4649>. - Zum aktuellen Geschehen am Horn von Afrika: Lisa Schlegel: Rezension zu: Walter Feichtinger, Gerald Hainzl, (Hrsg.): Somalia. Optionen - Chancen - Stolpersteine. Wien 2011, in: H-Soz-Kult, 21.03.2013, <www.hsozkult.de/publicationreview/id/rezbuecher-15807>. - Einen allgemeingefaßten Überblick vermittelt https://de.wikipedia.org/wiki/Deutsche Kolonialbestrebungen_an_der_Somaliküste [Dateneinsicht: 04.03.2018]. Zum Thema der Kolonialbestrebungen auch s. auch G. Ziethen (Anm. 19).

7 Lothar Dittrich, Annelore Rieke-Müller: Carl Hagenbeck (1844-1913). Tierhandel und Schaustellungen im Deutschen Kaiserreich. Frankfurt a.M u.a. 1998. S. 144-151. - Vgl auch die TV-Dokumentation unter dem Titel «"Die Wilden“ in den Menschenzoos». ARTE 28.09.2018, online zugänglich über die Mediathek des Senders ARTE https://www.arte.tv.Geschichte

$8 \quad$ Ebd. S. 37ff. - Vgl. auch Winfried Speitkamp: Rezension zu: Bernhard Gißibl: The Nature of German Imperialism. Conservation and the Politics of Wildlife in Colonial East Africa. New York 2016, in: H-Soz-Kult, 20.04.2017, <www.hsozkult.de/publicationreview/id/rezbuecher-26238>. 
und 1901 unterlagen, zumal sie auch über die englischen Kolonien angeworben werden konnten. ${ }^{9}$

Bekannt wurde Carl Hagenbeck indessen durch seinen Handel mit Straußen und der Aufnahme einer Straußenzucht von 1909 bis 1922. Speziell für die Beschaffung von Straußen war ein Tiersammler und Tierfänger (Josef Menges) beauftragt, von dem bekannt ist, daß er im Jahre 1879 vierzehn Tiere, im Jahre 1880 einunddreißig Tiere aus Nubien beschaffte. Josef Menges organisierte die Beschaffung seit 1885 eigenständig, die Lieferzahlen werden teilweise mit mehr als 70 Tieren genannt. Carl Hagenbeck zeigte Somali-Schauen 1882, 1885, 1894/95, wobei während einer Schau im Jahre 1885 einige Somalier auf Straußen durch Berlin ritten. Auch andere Zoos, Zirkusse sowie „Wandermenagerien“ gehörten zu Carl Hagenbecks Kunden. ${ }^{10}$ Wahrscheinlich war es dieses Umfeld, das Karl May zu seiner Erzählung anregte.

Josef Menges trat auch als Anwerber für die Hagenbeck'schen Völkerschauen auf. Später übernahm Walter Ebert diese Aufgabe; in zahlreichen Schauen wurden sie von einem somalischen Häuptling namens Herz(s)i Ergeh Gorseh unterstützt, der 1895 selbst in Begleitung seiner Stammesmitglieder nach Berlin kam. ${ }^{11}$ Die von Hilke Thode-Arora zusammengestellten Veranstaltungslisten erwähnen für die 1885 gezeigte Schau 15 Personen, für die in Basel gezeigte Schau des Jahres 1889, dem Erscheinungsjahr der beiden Erzählungen von Karl May: 16 Männer, 4 Frauen, 10 Jungen, 3 Mädchen aus Somalia. ${ }^{12}$

\footnotetext{
9 Dittrich/Rieke-Müller a.a.O. (Anm. 7) S. 171ff., 356 Abb. 40.

10 Ebd. S. 73ff., 209-213.

11 Ebd. S. 75.

12 Hilke Thode-Arora: Für fünfzig Pfennig um die Welt. Die Hagenbeckschen Völkerschauen. Frankfurt, New York 1989. S. 170-175, bes. 170f. Weitere Schauen mit Somaliern werden für die Jahre 1890/91, 1895, 1906, 1907, 1910/11, 1913/14, 1927 und 1929 genannt. Ab 1908/09 gibt es auch eigene Programme zum Thema „Äthiopien“. Vgl. Eric Ames: Carl Hagenbeck's Empire of Entertainments. Seattle/WA 2008. S. 30f., $132 \mathrm{f} \mathrm{zu}$ den Völkerschauen mit somalischen und äthiopischen Teilnehmern. - Zur Kulturgeschichte thematischer Ausstellungen: Stephanie Zehnle: Rezension zu: Jan-Erik Steinkrüger: Thematisierte Welten. Über Darstellungspraxen in Zoologischen Gärten und
} 
Im Verlauf dieser Schauen wurden nicht nur friedliche Lagerszenen dargestellt, sondern auch Kampf und Tod. ${ }^{13}$

Die von Karl May erwähnten persönlichen Kontakte zwischen Besuchern der Völkerschauen und deren Darstellern waren seitens der Veranstalter nicht gerne gesehen und man versuchte die mehrfach vorkommenden privaten Abendeinladungen, Liebesbeziehungen, Flirts, auch Eheschließungen, mit Anwerbeverboten zu verhindern (besonders 1901 infolge der Berliner Kolonialausstellung), da sich die Darsteller nach dem Ende der täglichen Veranstaltungen in den Städten frei bewegen konnten (und auch andere Existenzgrundlagen suchen konnten). Auch war man bemüht, Darsteller zu finden, die keine europäischen Verkehrssprachen sprachen. ${ }^{14}$

Die in der Erzählung „Sklavenrache“ erwähnten Ethnien der Schibril Abokr und Isa lassen sich nach der bei Musa Muhammad Omar 2002 erstellten Abstammungstabelle dem Ishaq-Clan, einem von insgesamt vier großen Clanfamilien der Somal, zuordnen, dessen Vorfahren der Legende nach von der arabischen Halbinsel stammen und dessen Nachfahren 1991 die Republik Somaliland (Hauptstadt Hargeisa) gründeten. ${ }^{15}$

\section{Rache und Vergebung}

Karl May wurde wahrscheinlich durch die Berichterstattung über die publikumswirksamen Veranstaltungen seiner Zeit dazu veranlaßt, sich

\footnotetext{
\begin{tabular}{lllll}
\hline Vergnügungsparks. & Bielefeld 2013, in: H-Soz-Kult, & 05.02.2015,
\end{tabular} <www.hsozkult.de/publicationreview/id/rezbuecher-22671>.

13 Vgl. Peer Zickgraf: Völkerschau und Totentanz. Deutsches (Körper-) Welttheater zwischen 1905 und heute. Marburg 2012. S. 51f.

14 Joachim Zeller: Von St. Pauli bis Hagenbeck: Völkerschauen in Hamburg. In: Ulrich van der Heyden, Joachim Zeller (Hrsg.): Kolonialismus hierzulande. Eine Spurensuche in Deutschland. Erfurt 2007. S. 330-336, bes. 335. - Vgl auch Thomas Morlang: Rezension zu: Zimmerer, Jürgen (Hrsg.): Kein Platz an der Sonne. Erinnerungsorte der deutschen Kolonialgeschichte. Frankfurt am Main 2013, in: H-Soz-Kult, 11.02.2014, <www.hsozkult.de/publicationreview/id/rezbuecher-21459>.

15 Musa Muhammad Omar: Ethnien und Nationalstaaten am Horn von Afrika. Somalia und Eritrea. Kulturelle Identität und politische Selbstbestimmung in der Weltgesellschaft 9. Münster./Westf. 2002. S. 95-99.
} 
entweder selbst als Zuschauer eine Vorstellung über den Ablauf zu verschaffen, oder er formte in der für seinen Schreibstil Vorgehensweise entsprechende Berichte um. Zur inhaltlichen Ausgestaltung des dargestellten Geschehens konnte er auf gedruckte Reise- und Expeditionsberichte zurückgreifen. Von diesen besaß er einige im Bestand seiner eigenen Bibliothek, dazu auch Wörterbücher.

Das 1931 publizierte Bibliotheksverzeichnis ${ }^{16}$ listet, abgesehen von dem im Text erwähnten Georges Révoil, folgende Bände auf, die in einem Zusammenhang mit dem Thema stehen.

Karl Andreae: Burton's Reise nach Medina und Mekka und in das Somaliland und Härrär in Ost-Afrika. Leipzig 1861.

Zur Sprache: Friedrich Müller: Über die Harrari-Sprache im östlichen Afrika. Wien 1863.

Da die beiden Zeitschriftenbeiträge „Das Straussenreiten der Somal“ und „Sklavenrache“ jedoch zum frühen Werk gehören, kann jedoch nicht mit Sicherheit gesagt werden, ob die genannte Literatur May schon zu diesem Zeitpunkt aus dem eigenen Bestand zur Verfügung stand, oder ob die Bücher erst später, als er wirtschaftlich arriviert war, angekauft wurden (was vielleicht wahrscheinlicher ist). Weil einige Erzählelemente auch im Roman „Die Sklavenkarawane“ (1889/90) im späteren Sudan-Zyklus ${ }^{17}$ „Der Mahdi“" (Trilogie, 1896) seines Werkes wieder auftauchen, war jedenfalls der Bedarf an zuverlässiger Hintergrundinformation gegeben.

Charakteristisch für diese frühe Werkphase ist das Motiv der Gefangenschaft (auch i.S. des Eingeschlossenseins bzw. der allgemeinen Abhängigkeit von oder Handlungsunfähigkeit in einer Situation) und der Rache. Dieser Zusammenhang wird in der wissenschaftlichen Literatur mit dem eigenen Erleben Mays erklärt, der in jungen Jahren aus materieller Not straffällig wurde. ${ }^{18}$ In der Aufarbeitung dieses Erlebens durchläuft Karl May als Mensch und als Autor eine tiefgreifende Wandlung von Rachegefühlen

\footnotetext{
16 Franz Kandolf/Adalbert Stütz/Max Baumann: Karl Mays Bücherei. Karl-May-Jahrbuch 1931, S. 212-291.

17 In Karl Mays Bibliothek befand sich der Band von Richard Buchta: Der Sudan und der Mahdi. Stuttgart 1884. Vgl. Kandolf u.a., op. cit. (Anm. 16), S. 228, 244. 
(weil er sich von der Gesellschaft ungerecht behandelt fühlte) hin zu Bedauern, Reue und pazifistisch geprägter Menschenliebe ${ }^{19}$. In seinem Beitrag „Die Rache und ihre Überwindung als Zentralmotiv bei Karl May ${ }^{\text {‘20 }}$ erwähnt Wolfgang Hammer 1994 eine weitere Kurzgeschichte Karl Mays, die inhaltlich dem Thema gewidmet ist. In der Kurzgeschichte „Die Rache des Mormonen“" $(1889 / 90)^{21}$ beschreibt Karl May eine aus Eifersucht und religiös konnotiertem Rassismus begangene, tödlich endende Beziehungstat eines fanatischen Mormonen, dessen Rache die unbeteiligten Mitglieder eines nordamerikanischen Indianerstammes zum Opfer fallen. Nur eine junge Häuptlingstochter, deren Ehre verletzt wurde, überlebt durch einen Zufall und in Unkenntnis der Ereignisse, die sich drohend abzuzeichnen beginnen. Die junge Frau flieht später in eine Stadt und erzählt dort von dem Geschehen. Danach verschwindet sie für immer. Diese Geschichte hat kein versöhnliches Ende. Anders dagegen in der Erzählung „Sklavenrache“, wo die jungen Überlebenden beschließen, den gegenseitigen $\mathrm{Haß}$ von Peinigern und Opfern zu überwinden.

Auch wenn sich in den Texten noch manche stilistischen Schwerfälligkeiten finden, die im späteren Werk Karl May nicht mehr auffallen, sind beide Erzählungen dennoch packend und wirken durch die Zuspitzung des unabwendbaren Unheils.

Zumindest im Kontext des Umgangs mit afrikanischen Sklaven dürfte sich Karl Mays Schilderung ebenfalls in einem historischen Rahmen bewegen. Insbesondere von den Aktivitäten, welche Sklavenhändler zur Zeit der Herrschaft Mehmed Alis (1769-1849, ab 1799 in Ägypten) von Ägypten aus in die südlichen Länder unternahmen, ist der rücksichtslose

\footnotetext{
19 Dazu vgl. Gabriele Ziethen: Mit Kara ben Nemsi und Graf Werniloff „In Mekka“. Gedanken zum Islamverständnis bei Karl May und Franz Kandolf. In: Wolfgang Biesterfeld u. Gabriele Ziethen: Geographia Poetica. Reisen zwischen Imagination und Realität. Interdisziplinäre Studien. Berlin 2018. S. 103-144, bes. 115, 118, 135, 136ff. mit 138 Anm. 124.

20 Wolfgang Hammer: Die Rache und ihre Überwindung als Zentralmotiv bei Karl May. Jahrbuch der Kar1-MayGesellschaft 1994. S. 51-84, bes. S. 70ff. gesellschaft.de/kmg/primlit/erzaehl/reise/mormone/mormonen.htm [Dateneinsicht: 04.03.2018].
} 
Umgang mit Sklaven afrikanischer Herkunft bekannt. ${ }^{22}$ Eine solche Sklavenjagd beschreibt Karl May ausführlich in seiner Reiseerzählung „Die Sklavenkarawane“ (Kap. 10 „In Sklavenfesseln“): das Aufsuchen geeigneter Dörfer, das Umstellen und Zerstören der Dörfer und das Selektieren der arbeitsfähigen Männer und jungen Frauen. Alle anderen werden getötet.

In der Zusammenschau zeigt sich, daß die Thematik und die Kritik an der Sklaverei ein wichtiges Thema in Karl Mays Schaffen war. Er dürfte damit zu den Autoren zu zählen sein, die in ihrer Zeit - es war ja auch die Zeit der europäischen Kolonialaktivitäten - Kritik an den entsprechenden Zuständen übte. Mit Blick auf das spätere Werk fällt auf, daß die auf die damaligen Leser unterhaltsam wirkende Thematik der „Völkerwiesen“ und „Völkerschauen“ zurücktritt und durch die literarische Ausgestaltung harter Realitätselemente im weiteren Verlauf des Werkes ersetzt wird. Karl May entwickelt schrittweise im Laufe seiner Romane eine ethische Grundhaltung, die mit der Kritik an bestehenden Zeitströmungen - hier Sklaverei, Kolonialismus und religiöser Fanatismus - einhergeht.

Angesichts moderner Formen von Sklaverei, Ausbeutung afrikanischer Arbeitskräfte, der anhaltenden Gewalt am Horn von Afrika und postkolonialer Überheblichkeit im Umgang mit den Ländern südlich der Sahara, sollten diese Texte neu entdeckt und interpretiert werden.

Anschrift der Autorin:

Dr. phil. Gabriele Ziethen M.A.

ziethen-aksum@t-online.de

www.gabrieleziethen.de

22 Einen drastischen Überblick über die Zusammenhänge von Machtentfaltung, Sklavenjagd und Handelswegen während des 19. Jhs. gibt Alan Moorehead: Zwischen Gott und Mohammed. Hundert Jahre Weltgeschichte am Nil. Stuttgart 1964. S. 169f. - Den Hinweis auf diesen Band verdanke ich Herrn Prof. Dr. Piotr O. Scholz, Schlangenbad (1943-2016). 